Nat Med 5:1256--1263, 1999 (November)

\title{
Chronic Brain Inflammation and Persistent Herpes Simplex Virus 1 Thymidine Kinase Expression in Survivors of Syngeneic Glioma Treated by Adenovirus-Mediated Gene Therapy: Implications for Clinical Trials
}

\section{Dewey RA, Morrissey G, Cowsill CM, Stone D, Bolognani F, Dodd NJ, Southgate TD, Klatzmann D, Lassmann H, Castro MG, Lowenstein PR}

The long-term consequences of adenovirus-mediated conditional cytotoxic gene therapy for gliomas remain uncharacterized. We report here detection of active brain inflammation 3 months after successful inhibition of syngeneic glioma growth. The inflammatory infiltrate consisted of activated macrophages/microglia and astrocytes, and T lymphocytes positive for leucosyalin, CD3 and CD8, and included secondary demyelination. We detected strong widespread herpes simplex virus 1 thymidine kinase immunoreactivity and vector genomes throughout large areas of the brain. Thus, patient evaluation and the design of clinical trials in ongoing and future gene therapy for brain glioblastoma must address not only tumor-killing efficiency, but also long-term active brain inflammation, loss of myelin fibers and persistent transgene expression.

PMID: 10545991, UI: 20015188

\section{Comments:}

This study addresses an issue relevant to all patients undergoing gene therapy in ongoing trials in which the investigators use the suicide gene herpes simplex virus--1 thymidine kinase (HSV-1-TK) system, with replication-incompetent retro- or adenoviral vectors. In experimental rodent models, this treatment has been remarkably successful in eradicating implanted gliomas. After viral infection and delivery of the suicide gene, ganciclovir treatment selectively kills transduced cells and nonspecifically damages other cells in the environment by the so-called "bystander effect." In the present study, Dewey and colleagues have observed the effects of ganciclovir treatment of adenoviral-delivered suicide in the rat CNS-1 tumor model. They noted significant inflammation in the brains of rats 3 months following a single dose the adenovirus/HSV-1-TK treated with ganciclovir. Although tumors were eliminated, extensive active inflammation was present, including demyelination. Furthermore, the transgene expression was detectable throughout the brain 3 months later.

What could be the mechanism for such chronic inflammation? Several possibilities are discussed by the authors. The first is that tumor antigen(s) released by the dying tumor cells invoke an immune response that is eliminated by macrophages. This is certainly the most desirable and tumor-specific possibility. However, the other possibilities include that the inflammation is vector induced (that is, nonspecific and 
diffuse in the brain); however, this is less likely because the chronic inflammation was not present in the hemisphere contralateral to where the tumor was implanted, although the vector expression was widespread. A third possibility is that the infection of macrophages themselves by the adenovirus could chronically stimulate antigen-specific T cells. A further possibility is underscored by the observation of synergy between the effect of ganciclovir and HSV-1-TK when given concurrently; this would imply that the immune response is secondary to cell killing in the process, and the bystander effect together with immunity directed against adenoviral antigens could be responsible.

Regardless of the mechanism involved, this paper conveys a cautionary note for patients enrolled in clinical trials in which this gene therapy is involved. The potential for nonspecific immune-mediated damage is present, which may have an impact on the functional neuronal circuitry surrounding the tumor cells. Such factors will likely become increasingly important in the future as survival in patients harboring malignant gliomas is extended with the identification of such novel targeting strategies.

William T. Couldwell, M.D., Ph.D. 